\title{
EL CENTRO DE INTERPRETACIÓN DEL CENTRO HISTÓRICO DE LIMA. ALCANCES Y PROYECCIÓN
}

\section{THE INTERPRETATION CENTER OF THE HISTORIC CENTER OF LIMA. SCOPE AND PROJECTION}

\author{
Natalia T. Deza de la Vega \\ Programa Municipal para la Recuperación del Centro Histórico de Lima (PROLIMA), Perú
}

\section{RESUMEN}

Con el objetivo de conocer la situación del Centro de Interpretación del Centro Histórico de Lima, su contexto, problemas y perspectivas, se realizó una investigación en la que se entrevistó a 32 personas y se efectuó un análisis FODA basado en la experiencia cotidiana y la revisión de documentos internos. Ello permitió recoger valiosa información sobre la percepción que tiene el público del Centro Histórico de Lima (CHL), sus limitaciones, las oportunidades y perspectivas para mejorar la gestión del Centro de Interpretación del Centro Histórico de Lima y el cumplimiento de sus objetivos, uno de los cuales es reforzar la identidad cultural de la población. Algunas de las recomendaciones, a partir de los resultados, son la presentación en inglés de los guiones museográficos, la mejora de la señalética, el establecimiento de un horario fijo de atención y la difusión por redes sociales en una plataforma propia.

Palabras clave: centro de interpretación, museografía, centro de visitantes, interpretación, actividades culturales.

\begin{abstract}
With the objective of knowing the situation of the Interpretation Center of the Historical Center of Lima, its context, problems and perspectives, a research was conducted in which 32 people were interviewed and a SWOT analysis based on daily experience and review of internal documents was carried out. This allowed gathering valuable information on the public perception of the Historical Center of Lima (CHL), its limitations, the opportunities and perspectives to improve the management of the Interpretation Center of the Historical Center of Lima and the fulfillment of its objectives, one of which is to reinforce the cultural identity of the population. Some of the recommendations, based on the results, are the presentation in english of the museum scripts, the improvement of the signage, the establishment of a fixed service schedule and the dissemination through social networks on their own platform.
\end{abstract}

Keywords: interpretation center, museography, visitors center, interpretation, cultural activities.

Este es un artículo Open Access bajo la licencia Creative Commons AtribuciónNoComercial-Compartirlgual 4.0

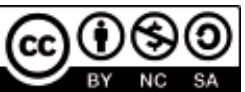


El Centro Histórico de Lima (CHL) es uno de los atractivos turísticos más visitados de la ciudad de Lima; su historia milenaria, caminos y canales, inmuebles virreinales y republicanos, centros culturales, museos, patios y balcones son parte de nuestro devenir como ciudad y son los que trasmiten su Valor Universal Excepcional. El CHL está inscrito desde 1991 como Patrimonio Mundial en la Organización de las Naciones Unidas para la Educación, la Ciencia y la Cultura (UNESCO).

[El Valor Universal Excepcional] significa una importancia cultural y/o natural tan extraordinaria que trasciende las fronteras nacionales y cobra importancia para las generaciones presentes y venideras de toda la humanidad. Por lo tanto, la protección permanente de este patrimonio es de capital importancia para el conjunto de la comunidad internacional. El Comité define los criterios de inscripción de los bienes en la Lista del Patrimonio Mundial. (UNESCO, 2008, p. 16)

A raíz de tal distinción, el 13 de julio de 1994, se creó el Programa Municipal para la Recuperación del Centro Histórico de Lima (PROLIMA), responsable de la protección, conservación y promoción del espacio en cumplimiento de la Convención sobre el Patrimonio Mundial Cultural y Natural que el Estado peruano suscribió en 1972 y el Congreso de la República del Perú ratificó en 1981. En años posteriores, 2011, 2013, 2015, UNESCO, mediante los informes de estado de conservación, indica como factor que afecta al CHL la interpretación y las facilidades en las visitas. ${ }^{1}$

En abril de 2017 se funda una sala temática en PROLIMA llamada Centro de Interpretación del Centro Histórico de Lima, con la finalidad de dar a conocer el patrimonio material e inmaterial del casco histórico de la ciudad y organizar eventos culturales con el mismo propósito. Desde esa fecha se realiza ahí una serie de actividades, sin costo alguno, dentro y fuera de su sala temática para generar la interacción con todo tipo de población; con los más jóvenes hasta con los adultos mayores.

Luego de más de dos años, desde su apertura, se considera conveniente realizar un análisis FODA, con el objetivo de conocer en qué medida el Centro de Interpretación del Centro Histórico de Lima está cumpliendo con sus objetivos, con respecto a los equipamientos culturales, los elementos museográficos, la publicidad de sus eventos, etc. Se plantea, en el presente trabajo, la necesidad de un centro de interpretación del CHL como espacio de trabajo y de experimentación, y para que la población se beneficie disfrutando de las actividades culturales que allí se realicen.

En principio, se desarrollan los conceptos interpretación y centro de interpretación; asimismo, se determinan los alcances y actividades del Centro de Interpretación del Centro Histórico de Lima; luego, se presentan los resultados de la aplicación de las entrevistas estandarizadas y del análisis FODA; y finalmente, las conclusiones y propuestas para mejorar su funcionamiento y lograr sus objetivos.

1 Respecto a este punto, las directrices prácticas para la aplicación de la Convención del Patrimonio Mundial del Comité del Patrimonio Mundial señala en el Formulario de propuesta de inscripción a la Lista del Patrimonio Mundial que la sección de servicios para visitantes y estadísticas se refiere a «servicios existentes in situ para visitantes, como por ejemplo explicaciones/interpretaciones, ya sea por medio de senderos, guías, anuncios o publicaciones; museo del bien, centro de visitantes 0 de interpretación; alojamiento; bares y restaurantes; tiendas; estacionamiento para vehículos; baños; servicio de investigación y rescate» (UNESCO, 2008, p. 119). 


\section{Definiciones}

El término interpretación está asociado a la historia de los parques naturales de Estados Unidos; surge a finales del siglo XIX como una actividad de comunicación y cultura. Los parques eran desconocidos para el gran público y quedaban en lugares agrestes y de difícil acceso, por lo cual, con el fin de recorrerlos, se necesitaba el acompañamiento de personas conocedoras del lugar, a quienes se les llamaba naturalistas; con el transcurso de los años el término varió a guías de naturaleza. Más adelante, con la profesionalización del oficio, no solo se trataba de guiar al visitante, sino también de difundir los valores del área (Ruiz, 2010, p. 166). En este sentido, se puede entender el término interpretar como explicar o declarar el sentido de algo.

La Asociación para la Interpretación del Patrimonio (AIP), con sede en España, señala en su sitio web que «la interpretación del patrimonio es el arte de revelar in situ el significado del legado natural y cultural al público que visita esos lugares en su tiempo libre» (2012). Si se desea conservar el patrimonio cultural se requiere de la interpretación para lograrlo porque la «interpretación es una actividad de comunicación diseñada para mejorar la calidad de la experiencia recreativa del visitante, y para inspirar, de una forma agradable, un mayor aprecio por el recurso» (Rideout-Civitarese, Legg y Zuefle, 1997, p. 27).

Por ende, la interpretación se dirige a las personas y a la sociedad en general con el fin de acercarlos al patrimonio cultural y/o natural de un lugar, ya sea a través de recorridos guiados, paneles, folletos u otros recursos. La interpretación tiene un propósito educativo, porque se muestra el significado del lugar para que el público usuario lo valore y comprenda, lo cual puede generar comportamientos positivos hacia el patrimonio que es visitado.

Complementando esta definición se hace hincapié en que la interpretación es una herramienta que puede tener la finalidad de preservar el patrimonio y gestionar los recursos patrimoniales promoviendo comportamientos positivos en los visitantes. No obstante, ello no suprime el hecho de que la información que alberga el centro de interpretación debe poseer «un mensaje claro, no denso ... reveladores de significados» como señala Morales Miranda en 1998 (Arcila Garrido, 2015, p. 147). Aunque el público no tenga la obligación de quedarse en el Centro de Interpretación del Centro Histórico de Lima, es conveniente considerar lo que señala Tilden (1977) sobre los visitantes: «quieren vagabundear o echar un vistazo, respirar profundamente, pasear, montar a caballo, hacer fotos, mezclarse con la gente haciendo todo eso, y olvidar su trabajo o existencia rutinaria».

Un centro de interpretación es también un espacio donde se exhibe un guion museográfico basado en los rasgos sobresalientes del patrimonio de un lugar, pero a diferencia del museo no necesita de objetos en el lugar, sino que busca comunicar su singularidad y valores. De acuerdo con Martín las funciones principales de un centro de interpretación son las siguientes:

Presentar un elemento patrimonial tanto natural como cultural exento del requisito de contacto directo con el recurso; dar claves suficientes para poder hacer comprensible el objeto patrimonial en cuestión; promover el uso y consumo de los productos típicos donde se ubica el centro de interpretación; generar deseos de conocer el territorio y todo lo que en él se encuentra. (2009, p. 53) 
La amplitud y complejidad del término centro de interpretación permite indicar que se puede crear un equipamiento interpretativo que forme parte de una gestión mayor, muchas veces, turística del patrimonio que se pueda desarrollar al interior y al exterior de un centro de interpretación. Asimismo, para Martín (2011) no existe normativa definida de este tipo de centros de interpretación, que muchas veces son llamados centros de visitantes, lugar de acompañamiento que resuelve las dudas del visitante con una atención personalizada.

El centro de interpretación explica lo que quiere cuidar por medio de una mediación directa o indirecta; directa cuando es con guías de turismo e indirecta mediante la exposición o exhibición (Alemán, 2014), este es el caso del Centro de Interpretación del Centro Histórico de Lima. En cambio los centros de visitantes, como señala Pierce (2004), buscan ser un espacio de orientación a los visitantes no solamente en su infraestructura, sino también en el trato con las personas para poder resolver sus preguntas y dudas.

\section{El Centro de Interpretación del Centro Histórico de Lima}

\section{Antecedentes}

En el Perú existen centros de interpretación referidos a lugares patrimoniales, por ejemplo, los de Cajamarquilla, Cieneguilla, Lomas de Lúcumo, Machu Picchu y Paracas, entre otros.

La temática predominante de los centros de interpretación que se encuentran en Lima está relacionada con los sitios arqueológicos ubicados cerca de los centros. El Complejo Arqueológico Monumental de Cajamarquilla se encuentra en el distrito de Lurigancho, Chosica; tiene la finalidad de brindar información sobre la importancia de la zona arqueológica e involucrar a los pobladores en la defensa y apropiación del patrimonio cultural de la zona, que fuera centro religioso y comercial construido de adobe.

Por su parte, el Centro de Interpretación Cultural de Cieneguilla, ubicado en plena plaza de armas del distrito cuenta con una explanada, una zona para el adulto mayor y zona de juegos para niños, lo que permite contar con gran afluencia de vecinos y visitantes. Realiza exposiciones culturales, simposios y talleres, y muestra cómo la zona de Cieneguilla formó parte del denominado Qhapac Ñam o Camino Inca.

Mientras que el Centro de Interpretación de las Lomas de Lúcumo se encuentra en Pachacamac, en el centro poblado rural Quebrada Verde. Se integra a la pendiente natural del terreno, ubicado en un ecosistema de lomas costeras, respetando la naturaleza de la zona. Posee espacios interactivos con tecnología audiovisual para conocer y aprender sobre la flora y fauna del ecosistema y la historia de Pachacamac.

El Centro de Interpretación del Centro Histórico de Lima se encuentra ubicado dentro del área declarada por la UNESCO como Patrimonio Mundial, reconocimiento otorgado en 1991 al mantenerse la integridad del conjunto en la zona declarada y la autenticidad y originalidad de los inmuebles y el trazado urbano. Cabe señalar que este centro forma parte del capítulo titulado Desarrollo Social del Plan Maestro del Centro Histórico de Lima al 2029 con visión al 2035, elaborado por PROLIMA y cuya aprobación aún está pendiente. ${ }^{2}$

2 El capítulo alude a la necesidad de la regeneración de las condiciones humanas para lograr una recuperación sostenible, integral, efectiva y permanente del Centro Histórico de Lima. 
El Centro de Interpretación del Centro Histórico de Lima empezó a diseñarse en la segunda mitad del año 2016. Se ubica en la propia sede de PROLIMA, en la cuadra 2 del Jirón. Áncash. ${ }^{3}$ El lugar donde se construyó la casa fue propiedad de Doña Gertrudis Querejazu, luego de Francisco Mendoza Ríos Caballero. En el siglo XX la casa fue hipotecada por el Banco Central Hipotecario del Perú hasta 1972. La Municipalidad de Lima recuperó la manzana del Rastro de San Francisco a partir del 2004 y la casa fue inaugurada en el año 2009 por el alcalde de entonces. Desde su apertura hasta mediados del 2018, el Centro de Interpretación del Centro Histórico de Lima funcionó en dos horario, de 9 a. m. a 1 p. m. y de 2 p. m. a 5 p. m., de lunes a viernes; desde agosto de 2018 se optó por un horario más extendido con el fin de facilitar la afluencia de visitantes.

Con la Resolución de Alcaldía n. ${ }^{\circ}$ 260, de marzo de 2019, se dispuso que el Programa Municipal para la Recuperación del Centro Histórico de Lima sea el órgano encargado de cumplir las funciones de Unidad Formuladora (UF) y Unidad Ejecutora de Inversiones (UEI) de la Municipalidad Metropolitana.

\section{Figura 1}

Lado izquierdo del Centro de Interpretación del Centro Histórico de Lima (julio 2019)

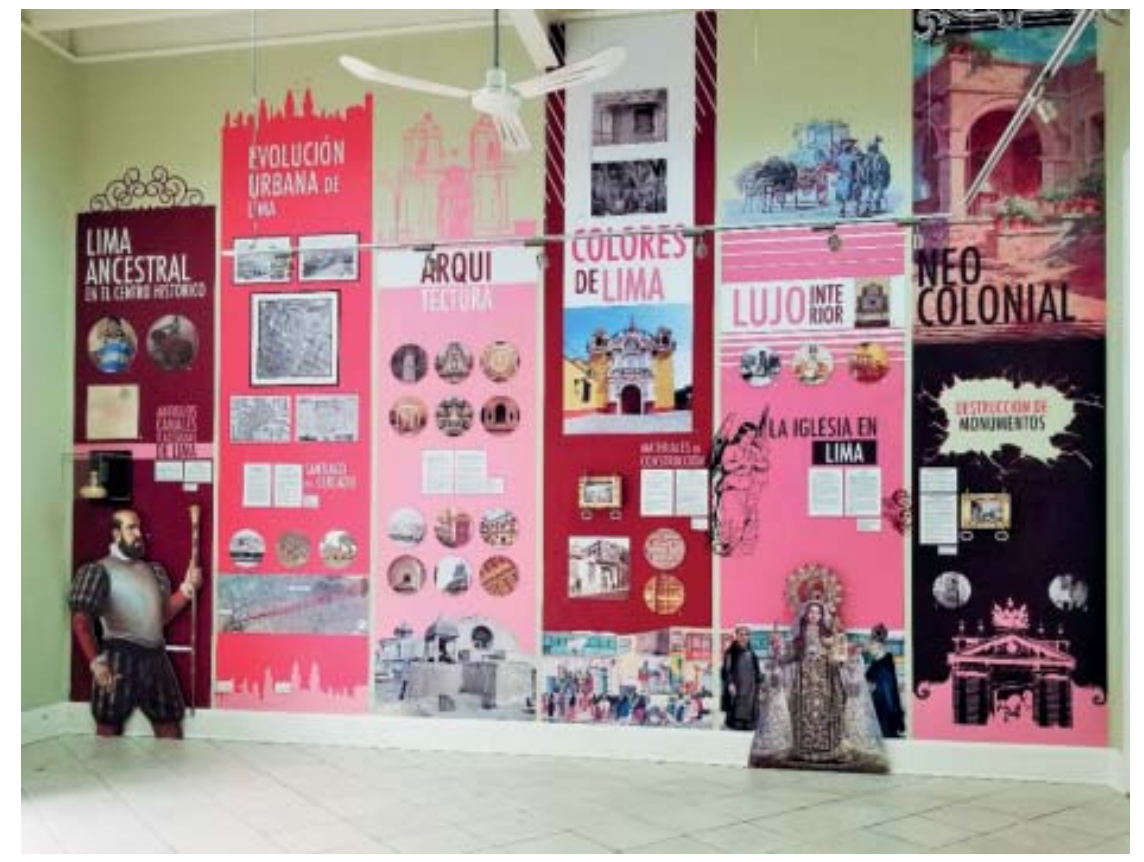

3 La sede de PROLIMA, en Jr. Áncash 229-239, Cercado de Lima, está considerada monumento histórico, construido a principios del siglo XX y restaurado a comienzos del siglo XXI. El Centro de Interpretación del Centro Histórico de Lima se encuentra en Jr. Áncash 239. Se llamó el Rastro porque en ella se encontraron los primeros camales o mataderos de la ciudad desde el siglo XVI. Allí se encuentra la sede del Museo de Sitio Bodega y Quadra. 


\section{Sistema de análisis}

\section{Entrevistas estandarizadas}

Con la finalidad de conocer las opiniones del público sobre el Centro de Interpretación del Centro Histórico de Lima se realizaron entrevistas estandarizadas, entre los meses de mayo y junio de 2019; estas incluyeron cinco preguntas, tres cerradas de opción múltiple y dos abiertas. La muestra no probabilística por conveniencia, la conformaron 32 personas que asistieron a las actividades de PROLIMA, tanto dentro como fuera del Centro de Interpretación del Centro Histórico de Lima.

Las edades de los entrevistados se encontraron entre los 22 y los 75 años, siendo del total 18 mujeres y 14 varones. En cuanto a la actividad principal que realizaban los entrevistados,13 señalaron ser trabajadores independientes, mientras que 19 ser jubilados o pensionistas o trabajadores dependientes.

Por último, se formularon dos preguntas abiertas que permitieron detectar aspectos que consideraban positivos, así como también aspectos por mejorar en el funcionamiento del Centro de Interpretación del Centro Histórico de Lima:

1. ¿Crees que el Centro de Interpretación del Centro Histórico de Lima beneficia a la ciudad?

2. ¿Cómo mejorarías el Centro de Interpretación del Centro Histórico de Lima?

Respondiendo a la primera pregunta, 29 personas indicaron que el Centro de Interpretación del Centro Histórico de Lima sí beneficia a la ciudad (tres personas no respondieron). Algunas de las razones brindadas fueron las siguientes:

- Definitivamente, todo espacio cultural donde se difunda el arte y la cultura con accesibilidad a la mayoría de la población, beneficia y contribuye con una mejor sociedad.

- Sí, para un limeño promedio que pasa obligatoriamente por el Centro de Interpretación del Centro Histórico de Lima para ir a su trabajo, centro de estudios, etc. y que lastimosamente desconoce del verdadero significado del patrimonio cultural que tenemos es conveniente la visita por este centro de interpretación, y para los turistas extranjeros es un plus al conocimiento incipiente que poseen de Lima prehispánica, virreinal y republicana.

Respecto a cómo mejorar el Centro de Interpretación del Centro Histórico de Lima dieron distintas respuestas, desde incrementar la difusión de las actividades, hasta mejorar la museografía con medios tecnológicos e involucrar a distintos públicos como niños, vecinos y estudiantes. Algunas de las respuestas fueron las siguientes:

- Colocando la información en inglés (o recurriendo a audios en inglés) y fomentando la visita de los turistas, con el fin de que puedan conocer la cultura limeña.

- Una forma sería tener un local más amplio para los talleres o charlas que realiza PROLIMA. Además, descentralizar los puntos de información para la captación de escolares, universitarios y público en general. 
- Incrementando la difusión por las redes sociales.

- $\quad$ Incluyendo sistema Braille, extendiendo el horario de atención.

\section{Análisis FODA}

Mediante este análisis se recogió información sobre los factores internos y externos que influyen en la marcha del Centro de Interpretación del Centro Histórico de Lima y sus proyecciones. Esta información permitió elaborar planes o estrategias para cumplir con los objetivos de la entidad. De este modo se detectaron las fortalezas y debilidades (factores internos) y las amenazas y oportunidades (factores externos) que se presentan en la matriz FODA (Tabla 1). El análisis se realizó entre los meses de mayo y junio de 2019, sobre la base de la experiencia cotidiana en el Centro de Interpretación del Centro Histórico de Lima y el estudio de documentos internos.

Tabla 1

Matriz FODA

Factores internos: fortalezas y debilidades

\section{Fortalezas}

- Entorno con gran riqueza cultural y patrimonial en el CHL.

- Espacio informativo que contribuye al reconocimiento del patrimonio cultural del CHL.

- Guion museográfico atractivo.

- Nuevas sedes del Centro de Interpretación del Centro Histórico de Lima

- Actividades sin costo alguno.

- Desarrollo de actividades con los vecinos y niños.

- Interés por parte de la gerencia de PROLIMA para mejorar sus servicios e introducir nuevos programas y/o proyectos.

- Tendencia ascendente en el número de visitantes desde el año 2017.

\section{Debilidades}

- Carece de señalética informativa para atraer o invitar a entrar a los visitantes.

- No siempre está abierto el Centro de Interpretación del Centro Histórico de Lima en el horario asignado.

- No se cuenta con una web o redes sociales propias.

- En el proyecto analizado de creación del Centro de Interpretación del Centro Histórico de Lima no se han contemplado los gastos de mantenimiento del espacio y de recursos humanos.

- No se tiene conocimiento del tipo de público que asiste al Centro de Interpretación del Centro Histórico de Lima.

- Carece de información en inglés (solamente la presentación).

- Espacio pequeño de trabajo.

- Carece de guías u orientadores.
Factores externos: oportunidades y amenazas

\section{Oportunidades}

- Lima es un lugar atrayente para los turistas y para el público en general.

- El sector declarado Patrimonio Mundial por la UNESCO que incluye al «Damero de Pizarro» contiene recursos materiales e inmateriales que transmiten el Valor Universal Excepcional del $\mathrm{CHL}$.

- La aprobación del Plan CHL.

- Posible inversión en las mejoras del Centro de Interpretación del Centro Histórico de Lima.

\section{Amenazas}

- La no continuación del Centro de Interpretación del Centro Histórico de Lima en una próxima gestión municipal.

- En términos de estadía Lima es un destino de tránsito y la de menor cantidad de noches.

- Gobierno nacional cierra constantemente calles. 


\section{Factores internos: fortalezas y debilidades}

La parte interna se refiere a las fortalezas y debilidades del Centro de Interpretación del Centro Histórico de Lima, es decir, a aspectos como el análisis del entorno, de los recursos humanos, análisis de actividades, entre otros.

\section{Fortalezas}

- $\quad$ Entorno con gran riqueza cultural y patrimonial en el CHL.

El Centro de Interpretación del Centro Histórico de Lima se encuentra ubicado en una zona delimitada y declarada Patrimonio Cultural de la Nación en 1972 e inscrita en la Lista de Patrimonio Mundial de la Unesco en 1991 por su valor universal excepcional, que se encuentra en el marco del cuarto criterio de selección para ser considerado Patrimonio Cultural de la Humanidad: «Ser un ejemplo sobresaliente de un tipo de edificio o de conjunto arquitectónico o tecnológico o de paisaje que ilustre una etapa significativa o etapas significativas de la historia de la Humanidad».

Según el Diagnóstico de Turismo que se incluye en el Plan Maestro del Centro Histórico de Lima al 2029 con visión al 2035 (Municipalidad Metropolitana de Lima [MML], 2019) se cuenta con 649 inmuebles declarados como Monumento Histórico, los cuales representan el 7.5\% de todos los inmuebles del CHL; además alberga colecciones muebles, que alcanzan 43690 bienes muebles inventariados en el SINAR ${ }^{4}$ como históricos, artísticos, etnográficos, arqueológicos e industriales y que incluyen pinturas, manuscritos, esculturas, textiles, libros, entre otros.

- Espacio informativo que contribuye al reconocimiento del patrimonio cultural del CHL.

El Centro de Interpretación del Centro Histórico de Lima es el primer espacio que se dedica íntegramente al CHL, es decir, no existe otro en la ciudad que se dedique solamente a ello.

El guion de la exposición permanente se elaboró a partir del aporte de 22 especialistas, entre historiadores, arquitectos y otros profesionales, a quienes se invitó a preparar breves artículos sobre temas relacionados con los valores culturales, arquitectónicos, históricos y religiosos de la ciudad de Lima (Tabla 2).

Todas las contribuciones fueron posteriormente recogidas en la publicación titulada Recuperando la Memoria presentada en julio de 2017 para el vigésimo tercer aniversario de PROLIMA.

- Guion museográfico atractivo.

La museografía de la sala del Centro de Interpretación del Centro Histórico de Lima, elaborada por el área de diseño de PROLIMA, está compuesta por paneles explicativos con imágenes troqueladas a todo color y con una gráfica explicativa que incluye los 22 temas mencionados en orden cronológico.

\footnotetext{
Siglas del Sistema de Registro Nacional Informatizado de Bienes Integrantes del Patrimonio Cultural de la Nación.
} 
Tabla 2

Temas de la sala de interpretación

- Lima ancestral

- Antiguos canales y acequias de Lima

- La reducción de Santiago del Cercado

- Evolución urbana de Lima

- La iglesia en Lima.

- Señor de los Milagros

- La Fiesta barroca

- La Semana Santa

- Arquitectura civil virreinal

- Arquitectura civil religiosa

- Acabados, colores y texturas de Lima
- Lujo, interior de inmuebles

- El estilo neocolonial

- Escuela limeña

- Costumbres gastronómicas

- Música virreinal

- La Lima de Ricardo Palma

- El folklore de Lima

- Textiles

- Limeños ilustres

- Las técnicas constructivas en la Lima virreinal y republicana

- Destrucción de inmuebles

Nota. Basado en PROLIMA, 2018

Entre los elementos que utiliza la exposición se encuentra una estación de sonido en la que se puede escuchar música barroca, y en otra música criolla. También se ha colocado un plano con ocho rutas patrimoniales ${ }^{5}$ elaboradas por PROLIMA, para su recorrido; para complementar, desde enero de 2017 hasta diciembre de 2018 se repartió folletería con las referidas rutas, especificando la historia del recorrido y la arquitectura de sus inmuebles. La investigación histórica y fotográfica del material fue elaborada por el equipo de historiadores y arquitectos de PROLIMA.

En la sala se ha usado un ceramio no original como parte del tema referido a la Lima ancestral en el CHL y un panel explicativo con una acuarela de Lima que señala los monumentos de las cinco rutas temáticas. Ahí mismo, al costado del plano de las rutas, se colocó el código de barras de la aplicación móvil diseñada en convenio con la Universidad Inca Garcilaso de la Vega y que contiene las ocho rutas patrimoniales.

Se han instalado tres cajas con fotografías y acuarelas que se pueden observar detenidamente utilizando manivelas para que avancen las imágenes. Hasta 2018 se contaba con dos cajas con reproductores de USB que incluían música barroca y música criolla. Por seguridad se retiraron las cajas.

- $\quad$ Nuevas sedes del Centro de Interpretación del Centro Histórico de Lima.

Téngase en cuenta que para los Juegos Panamericanos y Parapanamericanos la Municipalidad de Lima consideró necesario crear un pequeño centro de interpretación, denominado Casa Lima ${ }^{6}$, instalado a finales de julio de 2019 en el Parque de la Exposición para el público de la ciudad; contó con información sucinta sobre los 22 temas desarrollados en el Centro de Interpretación del Centro Histórico de Lima de PROLIMA,

5 Son cinco rutas temáticas y tres ejes patrimoniales. Las primeras incluyen recorridos por el Rímac, Monserrate, Barrios Altos y el Damero de Pizarro para revivir experiencias y explorar historia y temas sobre Lima. Los segundos están conformados por recorridos secuenciales por los jirones Áncash, Jirón de la Unión y Junín.

6 El diseño y los textos de investigación han sido elaborados por la gerencia de PROLIMA. 
con una instalación lúdica para hacer más entretenida la visita y con un sistema braille, método de lectura y escritura táctil diseñada para los invidentes.

Desde la Casa $\operatorname{Lima}^{7}$ se trazaron una serie de rutas a cargo de guías de turismo de la Municipalidad de Lima con el fin de recorrer el CHL. Entre las rutas elaboradas por la Subgerencia de Turismo de la Municipalidad Metropolitana de Lima están:

- Centro Histórico de espíritu bohemio, que invita a recorrer restaurantes, bares y lugares importantes para la ciudad.

- Barrio chino, recorrido por la conocida calle Capón en Barrios Altos.

- Palacios de Lima, reliquias culturales, recorrido por los palacios de la Plaza de Armas y otros ubicados en el CHL.

La propuesta de Casas Lima en otros lugares del CHL forma parte del capítulo Desarrollo Social del Plan Maestro del Centro Histórico de Lima, como una de las actividades de difusión del Programa de inducción, capacitación, divulgación del valor universal excepcional del CHL y fortalecimiento de la identidad cultural.

De otro lado, en julio de 2019 la Subgerencia de Turismo de la Municipalidad de Lima cambió el diseño de la Oficina de Información Turística, ubicada en el pasaje Nicolás de Ribera n. ${ }^{145}$, a la espalda de la Municipalidad de Lima e instaló un pequeño Centro de Interpretación del Centro Histórico de Lima.

- $\quad$ Actividades sin costo alguno.

El Centro de Interpretación del Centro Histórico de Lima organiza eventos gratuitos como parte del programa de difusión y promoción del CHL, entre ellos, presentaciones de libros, proyección de películas peruanas y coloquios. El bagaje histórico, cultural, arquitectónico y religioso de Lima es motivo de estudio para arqueólogos, historiadores, arquitectos y otros especialistas, por ello se creó el conversatorio LIMARTES, evento que se realiza el segundo martes de cada mes y que busca presentar nuevas investigaciones sobre la ciudad, con el fin de hacer más accesible su historia, patrimonio y recuperación a todos los ciudadanos.

Desde 2017 hasta julio de 2019 se han realizado 11 LIMARTES. En el año 2019 los LIMARTES se han realizado en distintos monumentos del Centro Histórico de Lima, y se han enfocado en temas relacionados con el Plan Maestro del Centro Histórico de Lima: Gestión del Riesgo de desastres para el Patrimonio Cultural, en la casa O‘ Higgins; el Paisajismo en el Centro Histórico de Lima, en la Casa Fernandini e Investigaciones arqueológicas en el Centro Histórico de Lima, en el Instituto Riva Agüero.

Es significativo anotar que el Centro de Interpretación del Centro Histórico de Lima se encuentra acompañado del Centro de Documentación del CHL que, inaugurado en el año 2016, tiene como objetivo custodiar y almacenar libros, material audiovisual, planos, documentos en formato físico y digital, así como promover y apoyar la investigación sobre la ciudad.

La Casa Lima será retirada al finalizar el certamen deportivo. 
- Desarrollo de actividades con los vecinos y niños.

Se han organizado 15 reuniones con los vecinos del CHL en el marco de las llamadas Jornadas sobre el Plan Maestro del Centro Histórico de Lima para difundirlo entre la población que reside y trabaja en el casco histórico de Lima. Las Jornadas, iniciadas a principios del año se han realizado en el Centro de Interpretación del Centro Histórico de Lima y en otros inmuebles del CHL. Son espacios de encuentro, de comunicación y de relación con vecinos, comerciantes y autoridades vecinales del casco histórico para promover la participación ciudadana y generar procesos de reflexión de este plan maestro.

Asimismo, con el fin de desarrollar la creatividad de los más pequeños, se ha programado, para la segunda mitad del año 2019, realizar actividades con niños que estudian en el CHL. Estas actividades comprenden la narración de cuentos sobre el CHL y talleres de dibujo y pintura; el Centro de Interpretación del Centro Histórico de Lima, con estas actividades tiene por objetivo fortalecer la identidad cultural de los niños para que entiendan, valoren y se identifiquen con su ciudad.

- Interés por parte de la gerencia de PROLIMA para mejorar sus servicios e introducir nuevos programas y/ o proyectos.

Desde mediados del año 2019 se ha reducido el espacio al contar con nuevos empleados en las oficinas de la gerencia de PROLIMA, a pesar de ello, se ha considerado necesario mantener el área del Centro de Interpretación del Centro Histórico de Lima como tal y abrirlo para los visitantes del CHL. Asimismo, la gerencia ha planteado mejorar el guion museográfico con paneles en escritura Braille y en inglés.

- $\quad$ Hay una tendencia ascendente en el número de visitantes desde el año 2017.

Con respecto al número de asistentes a las actividades del Centro de Interpretación del Centro Histórico de Lima desde su creación hasta junio de 2019, la cifra es de 107682 personas. Esta cifra se conoce debido a la instalación de un contador mecánico y a las listas de asistentes a los eventos organizados por el Centro de Interpretación del Centro Histórico de Lima, tanto dentro como fuera del local (ver Tabla 3).

\section{Debilidades}

- Carece de señalética informativa para atraer o invitar a entrar a los visitantes.

Hasta finales del año 2018 se contaba con un banner ubicado a la entrada del Centro de Interpretación del Centro Histórico de Lima, el cual señalaba solamente el nombre de la institución y la gerencia, el horario de atención, números telefónicos, correo electrónico y dirección.

- $\quad$ No siempre está abierto el Centro de Interpretación del Centro Histórico de Lima en el horario asignado.

El horario regular del Centro de Interpretación del Centro Histórico de Lima es de lunes a viernes, de 9 a. m. a 1 p. m. y de 2 p. m. a 7 p. m.; también abrió sus puertas en días especiales para la ciudad como por ejemplo, Semana Santa y los domingos en el marco proyecto Al Damero de Pizarro sin carro de la Municipalidad de Lima, el cual promueve la participación familiar para realizar diversas actividades culturales 


\section{Tabla 3}

Asistencia a las actividades del Centro de Interpretación del Centro Histórico de Lima, desde el 2017 al 2019

\begin{tabular}{|c|c|c|c|c|}
\hline \multirow[b]{2}{*}{ MESES } & \multicolumn{3}{|c|}{ AÑO } & \\
\hline & 2017 & 2018 & 2019 & \\
\hline ENERO & & 2515 & 6295 & \\
\hline FEBRERO & & 2898 & 5910 & \\
\hline MARZO & & 8691 & 3418 & \\
\hline ABRIL & 7316 & 3467 & 3796 & \\
\hline MAYO & 3714 & 3338 & 4197 & \\
\hline JUNIO & 3360 & 3740 & 3883 & \\
\hline JULIO & 3153 & 5293 & & \\
\hline AGOSTO & 2091 & 4828 & & \\
\hline SETIEMBRE & 2820 & 3455 & & \\
\hline OCTUBRE & 3286 & 3357 & & \\
\hline NOVIEMBRE & 2312 & 3874 & & \\
\hline DICIEMBRE & 2269 & 4406 & & \\
\hline TOTALES & 30321 & 49862 & 27499 & 107682 \\
\hline
\end{tabular}

Nota. Basado en PROLIMA, 2019

que han permitido atraer a gran cantidad de visitantes al Centro de Interpretación del Centro Histórico de Lima. $^{8}$

En la actualidad el Centro de Interpretación del Centro Histórico de Lima presta sus ambientes fuera de horas de trabajo a una asociación de cantantes de ópera. ${ }^{9}$ Además, se realizan actividades de difusión sobre el CHL y reuniones de trabajo entre los integrantes de la gerencia de Prolima y/o con otros miembros de la MML y otras instituciones.

Cabe señalar que la Subgerencia de Turismo de la Municipalidad de Lima desde 2017 presenta en el Centro de Interpretación del Centro Histórico de Lima las charlas Gestores Turísticos para dar a conocer a profesionales y público en general los atractivos culturales que se encuentran en Lima. Sin embargo los horarios reducidos limitan las opciones de los visitantes.

- $\quad$ No se cuenta con un sitio web o redes sociales propias.

La difusión del Centro de Interpretación del Centro Histórico de Lima se realiza a través del sitio web y el Facebook de la Municipalidad de Lima porque no posee un medio oficial propio para difundir sus actividades. Pero sí existe una página web, que fue subida a la nube luego de inaugurado el Centro de Interpretación del Centro Histórico de Lima y que ofrece una vista en 360 grados de la sala.

El enlace es https://www.360cities.net/image/centro-de-interpretacion-prolima-1

8 El Plan Maestro del Centro Histórico de Lima al 2029 con visión al 2035 está incluyendo un Calendario de la Identidad de la ciudad, el cual posee fechas importantes y tradicionales de la ciudad como el carnaval y la Semana Santa para celebrarlas como parte de las actividades de la Municipalidad de Lima. Ello servirá para que más turistas asistan al casco histórico para las celebraciones previstas en el calendario.

$9 \quad$ PROLIMA tiene previsto ofrecer conciertos o recitales de música con la asociación en el año 2019. 
Se han elaborado notas de prensa sobre las actividades del Centro de Interpretación del Centro Histórico de Lima que han aparecido en diarios online como TVRobles y Lucidez. Son pocas las veces que se han difundido las actividades en prensa escrita. La difusión, sobre todo, se ha realizado por medio de páginas de Facebook que difunden actividades del CHL. Asimismo, se dan a conocer mediante el envío de correos a las personas que asistieron a las actividades de PROLIMA ${ }^{10}$ como de post informativos a cuentas de Facebook que difunden actividades culturales en Lima.

- En el proyecto analizado de creación del Centro de Interpretación del Centro Histórico de Lima no se han contemplado los gastos de mantenimiento del espacio y de recursos humanos.

La creación del Centro de Interpretación del Centro Histórico de Lima ha significado una inversión monetaria en la museografía, en herramientas de trabajo para eventos como ecran y sillas. En julio de 2019 se ha solicitado la adquisición de un nuevo proyector, puntero laser, ecran, nuevos focos led, parlante y micrófono. Por otra parte, el personal de limpieza diariamente limpia el espacio. Cabe indicar que no se ha realizado ningún mantenimiento especial del Centro de Interpretación del Centro Histórico de Lima porque se encuentra en buen estado.

- No se tiene conocimiento del tipo de público que asiste al Centro de Interpretación del Centro Histórico de Lima.

No se ha hecho un análisis del público que asiste al Centro de Interpretación del Centro Histórico de Lima y del público susceptible de ser atraído al centro.

- $\quad$ Carece de información en inglés.

Aunque el Centro de Interpretación del Centro Histórico de Lima cuenta con una presentación en castellano y en inglés sobre la creación e importancia del centro, los textos del guión museográfico solo están en castellano y abarcan los 22 temas ubicados en los paneles. No hay otro elemento que integre el sistema de interpretación patrimonial del centro para turistas con dominio del idoma inglés.

- $\quad$ Espacio pequeño de trabajo.

EL Centro de Interpretación del Centro Histórico de Lima ocupa una sala con un área de $52 \mathrm{~m}^{2}$ en el primer piso del inmueble, donde se ubica la gerencia de PROLIMA. Es un ambiente pequeño que sirve como sala de reuniones y sala de exposición. Además, se encuentran el área de archivo administrativo e histórico, un área de conservación, así como el centro de documentación.

- $\quad$ Carece de guías u orientadores.

El Centro de Interpretación del Centro Histórico de Lima no cuenta con guías en la actualidad. Cuando se abrió en el año 2017 se contaba con voluntarios que apoyaban en la orientación a los turistas que ingresaban al espacio. Este centro busca ser un punto de apertura para las personas que recorran el CHL al cubrir con 22 temas lo que se puede encontrar o conocer dentro del casco histórico de la ciudad.

10 Con el fin de fidelizar al público que ya conoce PROLIMA y las actividades del Centro de Interpretación del Centro Histórico de Lima. 
De manera temporal, desde julio de 2019 hay un especialista de la Subgerencia de Turismo de la MML que brinda información a los turistas; el nuevo integrante del Centro de Interpretación del Centro Histórico de Lima cuenta con un stand con información de la ciudad, sobre rutas a la carta en el CHL y material informativo sobre otras ciudades del Perú. El puesto turístico en el Centro de Interpretación del Centro Histórico de Lima fue colocado como punto de referencia para los visitantes que llegaban a Lima con motivo de los Juegos Panamericanos y Parapanamericanos.

\section{Factores externos}

El sector externo se enfocó en las oportunidades que ofrece el entorno, el CHL y la ciudad. Las amenazas presentadas son las circunstancias en las que el Centro de Interpretación del Centro Histórico de Lima encuentra dificultad para alcanzar una mejor gestión del mismo.

\section{Oportunidades}

- Lima es un lugar atrayente para los turistas y para el público en general.

Lima recibe el 90\% de los visitantes que llegan al Perú y que pasan al menos una noche en la capital peruana. Solamente entre enero y abril de 2019 se han recibido 1463499 turistas; de estos, el 55\% (809 794 turistas) tuvo a Lima como punto de ingreso (Cusirramos, 2019).

Es preciso indicar que, al Aeropuerto Internacional Jorge Chávez, llegan principalmente visitantes de los Estados Unidos, que es a su vez, el segundo país emisor de turistas al Perú, luego del país vecino Chile.

De otro lado, gracias a su ubicación en el centro de la ciudad y a la ubicación de oficinas gubernamentales, servicios y lugares comerciales que posee, atrae a la población diariamente, población flotante que, según INEI, se estima son alrededor de 1.5 a 2 millones de personas cada día (citado en Plan Maestro del CHL al 2029 con visión al 2035). Asimismo, los residentes de Lima Metropolitana en encuestas de los años 2015 y 2016 realizadas por Lima Cómo Vamos (2017) tienen al Cercado de Lima como destino principal de los viajes para ir a trabajar y/o estudiar.

- El sector declarado Patrimonio Mundial por la UNESCO que incluye al Damero de Pizarro ${ }^{11}$ contiene recursos materiales e inmateriales que transmiten el valor universal excepcional del CHL.

EL Plan Maestro del Centro Histórico de Lima forma parte del área designada como Patrimonio Cultural de la Nación desde 1972 y Patrimonio Mundial desde 1991. Existe patrimonio cultural material como iglesias con bienes muebles patrimoniales, monasterios y conventos, balcones El patrimonio cultural inmaterial con el que cuenta el CHL incluye fiestas religiosas como la del Señor de los Milagros que tiene un origen virreinal; otro ejemplo es la producción de dulces tradicionales en el Monasterio del Carmen Alto en Barrios Altos, entre otros.

11 El sector al que se hace referencia es la configuración fundacional de la ciudad desde 1535, es decir la Lima en cuadrículas que se centra alrededor del actual Palacio de Gobierno. 
- La aprobación del Plan Maestro del Centro Histórico de Lima. ${ }^{12}$

Es muy probable que para fines del año 2019 se pueda tener aprobado el Plan Maestro del Centro Histórico de Lima lo cual permitiría aplicar el capítulo sobre Desarrollo Social que incluye más labores con la población del CHL y el Plan Maestro del Centro Histórico de Lima.

- Posible inversión en las mejoras del Plan Maestro del Centro Histórico de Lima.

La gestión de la gerencia de PROLIMA tiene una serie de prioridades referidas al Plan Maestro del Centro Histórico de Lima, como la difusión del mismo y de sus actividades inmediatas a realizar después de su aprobación. El Plan Maestro del Centro Histórico de Lima no solamente es al 2029, lo que incluye recibir el bicentenario con una recuperación de la ciudad sino que tiene como visión el 2035.

\section{Amenazas}

- La no continuación del Centro de Interpretación del Centro Histórico de Lima en una próxima gestión municipal.

El inmueble de la gerencia de PROLIMA actualmente se encuentra al tope de personal. Es posible que la próxima gestión de PROLIMA quiera ocupar ese espacio como oficina o almacén.

- En términos de estadía, Lima es un destino de tránsito y la de menor cantidad de noches de alojamiento invertidas.

Las personas que visitan la ciudad de Lima como turistas prefieren visitar Miraflores, Barranco o San Isidro, en vez de visitar el CHL. El tráfico de la ciudad se ha vuelto muy difícil y caótico lo que no favorece las visitas al CHL.

- Gobierno nacional cierra constantemente calles.

En el Damero de Pizarro existen varias instituciones de gobierno como el Ministerio de Economía, la Presidencia del Consejo de Ministros, el Tribunal Constitucional y el Palacio de Gobierno, la mayoría de ellas recibe manifestaciones públicas a lo largo del año, debido a eso los gobiernos de turno optan por cerrar las calles aledañas, evitando así que los manifestantes se acerquen, sobre todo, a Palacio de Gobierno. El acceso por el Jr. Carabaya cuadra 1 no es restringido, ello facilita que siempre transiten personas por el Jr. Áncash cuadra 2.

Con esta evaluación se determinan los aspectos positivos y negativos del Centro de Interpretación del Centro Histórico de Lima y las estrategias de actuación.

12 El 5 de diciembre de 2019 el Concejo Metropolitano de Lima aprobó el Plan Maestro del Centro Histórico de Lima al 2029 con visión al 2035 y su Reglamento Único de Administración con la Ordenanza n. ${ }^{\circ} 2194$ y la Ordenanza n. 2195 respectivamente. 


\section{Resultados}

Mediante las entrevistas a 32 personas se ha determinado la situación del Centro de Interpretación del Centro Histórico de Lima, así como la importancia de contar con este establecimiento en el centro histórico de la ciudad. Aunque es una muestra intencional presenta datos interesantes que se han tomado en cuenta para esta investigación, validos para complementarlos con el análisis FODA.

Precisamente, el análisis FODA, instrumento viable para analizar cómo se labora en el Centro de Interpretación del Centro Histórico de Lima, si bien no identifica todas las variables, sí brinda una descripción analítica que permite definir la situación y recomendar cambios. Asimismo, da cuenta, en lo que respecta a las fortalezas, que el espacio logra formar parte de un sector muy transitado por población flotante y por turistas; y al realizar actividades sin costo alguno facilita la accesibilidad a dichos eventos por parte de niños, vecinos y público en general.

El Centro de Interpretación del Centro Histórico de Lima detenta un guion museográfico atractivo lo que ha permitido ser aprovechado por la propia MML para que sea referente en otros dos espacios importantes del CHL.

Respecto a las debilidades, se presentan ocho, que pueden significar un reto en el trabajo diario. El público que visita el Centro de Interpretación del Centro Histórico de Lima se puede acercar al Centro de Documentación del CHL para consultar sobre temas históricos, solicitar material documental y/o material fotográfico, ubicación de monumentos y museos, entre otros.

No aparecer en redes sociales ${ }^{13}$ propias dificulta tener una comunicación instantánea para compartir información y conocimientos de actividades y funciones. No obstante, gracias al Plan Maestro del Centro Histórico de Lima se está dando a conocer con mayor detalle la gerencia de PROLIMA teniendo más oportunidades de aparecer en medios de comunicación masiva como la radio y la televisión.

La carencia de una señalética apropiada en la entrada del local, no poseer información en inglés y no tener un horario fijo de apertura, reducen la atención y el interés de los turistas por el Centro de Interpretación del Centro Histórico de Lima. Sin embargo, también son una oportunidad para entablar diálogo con las asociaciones de guías de turismo para dar a conocer el espacio, explicar los temas que se desarrollan y que así ellos puedan visitarlo en su tránsito cotidiano y mostrar a los turistas los monumentos o espacios públicos del CHL. Es un aporte para que los guías tengan la posibilidad de explicar en inglés y otros idiomas las diversas materias que desarrolla el Centro de Interpretación del Centro Histórico de Lima.

Respecto a las amenazas, la no continuidad del Centro de Interpretación del Centro Histórico de Lima, es un riesgo que puede ocurrir en una próxima gestión municipal; las visitas al CHL pueden decrecer aún más por el tránsito en la ciudad y porque cierran las calles frecuentemente.

13 Para saber la importancia de las redes sociales se puede aludir el estudio de Alvarado Peña respecto a los medios que emplean las diversas organizaciones (centros culturales, galerías y museos de Lima metropolitana) para comunicarse con su público; en dicho estudio se encontró que el $82 \%$ de las instituciones encuestadas señala que «Facebook es su medio predilecto y más efectivo, ya que lo consideran de fácil uso, barato y que genera el impacto deseado, permitiéndoles establecer un contacto directo con su público» (Alvarado Peña, 2018, p. 93). 
El modelo FODA se ha complementado con el análisis de las funciones del Centro de Interpretación del Centro Histórico de Lima que forman parte del plan de acción anual de PROLIMA, que incluye entre sus tareas la que se menciona en la cuarta función, consistente en la Difusión y promoción cultural del CHL.

Son funciones del Centro de Interpretación del Centro Histórico de Lima:

1. $\quad$ Recibir y orientar a los visitantes y habitantes para que aprendan, comprendan y disfruten el CHL desde una visión multidimensional.

2. $\quad$ Educar, motivar e incentivar el aprendizaje entre los visitantes, ayudándolos a investigar sobre los valores materiales e inmateriales del CHL.

3. Desarrollar proyectos de investigación y fortalecimiento de la identidad social y cultural, promovidos por PROLIMA.

4. Difundir y realizar eventos y actividades culturales relacionadas a la historia y patrimonio del CHL.

5. $\quad$ Ser un punto de encuentro para grupos de especialistas sobre el CHL donde se promueva la investigación y el planteamiento de nuevos proyectos e iniciativas.

6. Capacitar a los agentes culturales que trabajan en la difusión del CHL, organizando actividades de capacitación y distribución de material informativo.

7. Recoger y analizar información acerca de los temas de interés del público en general sobre el patrimonio del centro histórico de Lima. (PROLIMA, 2018, p. 97)

Sobre la primera función, se debe indicar que a pesar de no contar con una persona permanente que atienda el Centro de Interpretación del Centro Histórico de Lima, las personas se pueden acercar al Centro de Documentación del CHL para hacer consultas. Sobre la segunda función, se llega a cumplir en el Centro de Interpretación del Centro Histórico de Lima con los talleres y conferencias dentro y fuera del espacio; la tercera función depende de la inclusión de un capítulo sobre desarrollo social en el Plan Maestro del Centro Histórico de Lima.

Con respecto a la cuarta función, PROLIMA cumple con realizar estas actividades desde su fundación en abril de 2017; la quinta función sí llega a cumplirse en PROLIMA porque el Centro de Interpretación del Centro Histórico de Lima realiza actividades y talleres de patrimonio donde congregan a arquitectos, guías de turismo, historiadores, entre otros; respecto de la sexta función se debe señalar que a raíz de la consulta pública del Plan Maestro del Centro Histórico de Lima se han realizado talleres informativos a guías de turismo y a la propia Subgerencia de Turismo de la Municipalidad Metropolitana de Lima sobre el capítulo del Plan Maestro del Centro Histórico de Lima que contempla un plan de turismo en el CHL. Finalmente la séptima función se cumple cuando se conversa con el público asistente para conocer sus intereses, y así, mejorar el trabajo realizado. 


\section{Recomendaciones}

El Centro de Interpretación del Centro Histórico de Lima es una entidad que beneficia a la ciudad, por lo tanto, su difusión por redes sociales es necesaria, así como la presentación de información en idioma inglés.

En el análisis FODA se observa que el espacio forma parte de un sector muy transitado por población flotante y por turistas. Por ello, es conveniente conocer los diversos intereses de los visitantes, lo que se puede hacer en el momento que se registran para ingresar a las actividades del Centro de Interpretación del Centro Histórico de Lima; información de gran utilidad para la programación de próximas actividades.

Así mismo, es importante brindar información acerca de las actividades gratuitas del Centro de Interpretación del Centro Histórico de Lima, que se vienen programanado con mayor frecuencia bajo la responsabilidad del Plan Maestro del Centro Histórico de Lima y LIMARTES.

Respecto a las debilidades, se presentan ocho, que pueden significar un reto en el trabajo diario del Centro de Interpretación del Centro Histórico de Lima. El público que visita el Centro de Interpretación del Centro Histórico de Lima se puede acercar al Centro de Documentación del CHL para efectuar las consultas. La falta de una señalética a la entrada del Centro de Interpretación del Centro Histórico de Lima resta un poco de interés por el espacio ubicado en Jr. Áncash cuadra 2. Se debe diseñar un banner informativo (en inglés y castellano) con datos sobre el Centro de Interpretación del Centro Histórico de Lima para interesar al visitante de la ciudad; un banner informativo para cada evento, también es otra alternativa.

La falta de un horario determinado para la apertura del Centro de Interpretación del Centro Histórico de Lima constituye un gran problema. Para superar esta debilidad es necesario fijar un cronograma mensual de actividades dentro del Centro de Interpretación del Centro Histórico de Lima, a partir del cual se pueda establecer horarios reales y días de acceso para el público. En este sentido, se podría optar por dejar de efectuar actividades a puerta cerrada en el Centro de Interpretación del Centro Histórico de Lima los días lunes, ya que es el día en que muchos centros culturales o museos permanecen cerrados.

En el presupuesto anual de la gerencia de PROLIMA es necesario incluir una partida exclusiva para los gastos de mantenimiento y de recursos humanos del Centro de Interpretación del Centro Histórico de Lima, considerándola como medida a largo plazo y no urgente.

Es imprescindible que la gerencia de PROLIMA cuente con redes oficiales como Facebook e Instagram para lograr una comunicación más rápida y fluida con los visitantes; esto no solo permitirá conocer las necesidades de los que asisten al espacio, sino mejorar los servicios del Centro de Interpretación del Centro Histórico de Lima.

Finalmente, para la planificación de actividades en el Centro de Interpretación del Centro Histórico de Lima es necesario considerar la diversidad del público, temas de interés y datos para contactarlos y enviarles información. 


\section{Conclusiones}

Esta investigación se basa en el trabajo realizado mediante entrevistas, así como en el estudio de las fortalezas, amenazas, oportunidades y debilidades (análisis FODA) del Centro de Interpretación del Centro Histórico de Lima.

Se establecen las definiciones y alcances de los términos interpretación, centro de interpretación y centro de visitantes. Además, se realiza una descripción del Centro de Interpretación del Centro Histórico de Lima que permite plantear recomendaciones para mejorar las funciones del mismo y contribuir al logro de sus objetivos.

A pesar que la infraestructura del Centro de Interpretación del Centro Histórico de Lima es pequeña y que PROLIMA no cuenta con otro ambiente propio para realizar actividades, se logra establecer un diálogo con los vecinos y turistas mediante la interpretación del patrimonio cultural de la ciudad, así como también reforzar la identidad cultural de la población.

El Centro de Interpretación del Centro Histórico de Lima trasmite una sola historia o mensaje referido al valor universal excepcional del Centro Histórico de Lima porque presenta los temas que caracterizan y hacen único a este patrimonio mundial. Además, se cumple con el requerimiento de la UNESCO y cuenta con el respaldo de las autoridades locales, siendo ello una condición para que pueda constituirse como un punto turístico de mucha importancia. 


\section{Referencias}

Alemán, A. (2014). Reflexiones sobre la Interpretación del Patrimonio. Revista de la Asociación de Docentes de la USMP, 28. http:// www.revistacultura.com.pe/revistas/RCU_28_1_reflexiones-sobre-la-interpretacion-del-patrimonio.pdf

Alvarado Peña, E. (2018). Hacia un museo sostenible: Oferta y demanda de los museos y centros expositivos de Lima. Lima: Museo de Arte de Lima.

Arcila Garrido, M. y López Sánchez, J. A. (2015). Los centros de interpretación como motor de desarrollo turístico local, ¿un modelo fracasado? El caso de la provincia de Cádiz. Boletín de la Asociación de Geógrafos Españoles, 67, 143-165. https://dialnet.unirioja.es/ servlet/articulo?codigo=5035571

Asociación para la Interpretación del Patrimonio (AIP). (2012). La AIP. https://www.interpretaciondelpatrimonio.com/es/la-aip

Cusirramos, C. (2019). ¿Cómo le fue al turismo a inicios del 2019? https://www.promperu.gob.pe/TurismoIN/sitio/ VisorDocumentos?titulo=\%C2\%BFC\%C3\%B3mo\%20le\%20fue\%20al\%20turismo\%20a\%20inicios\%20del\%202019?\&url=/Uploads/ infografias/1075/TurismoMundoPeruICuatrim2019.pdf\&nombObjeto=Infograf\%C3\%ADas\&back=/TurismoIN/sitio/ Infografias\&issuuid $=0$

Lima cómo vamos. (2017). VII Informe de percepción sobre calidad de vida en Lima y Callao. http://www.limacomovamos.org/cm/ wp-content/uploads/2017/04/EncuestaLimaC\%C3\%B3moVamos_2016.pdf

Martín, P. C. (2009). Los centros de interpretación: urgencia o moda. Hermes, 1. https://core.ac.uk/download/pdf/83011769.pdf

Martín, P. C. (2011). Estudio analítico descriptivo de los centros de interpretación patrimonial en España [tesis doctoral, Universidad de Barcelona]. Barcelona, España.

Pierce, P. L. (2004). The Functions and Planning of Visitor Centres in Regional Tourism. The Journal of Tourism Studies,15(1). https:// pdfs.semanticscholar.org/be16/982fdd392aef3eae437bedaf5edfe0a6d287.pdf

PROLIMA. (2018). Memoria de PROLIMA 2015 a 2018. Municipalidad Metropolitana de Lima, Perú.

PROLIMA. (2019). Plan Maestro del Centro Histórico de Lima al 2029 con visión al 2035. http://imp.gob.pe/images/ Plan\%20Maestro\%20del\%20Centro\%20Historico/II.\%20Diágnostico/01\%20Diágnostico/DIAGNOSTICO.pdf

Rideout-Civitarese, S., Legg, M. H. y Zuefle, D. M. (1997). More Thoughts on the difference between environmental interpretation and environmental education. Legacy: The magazine of the National Association for Interpretation, 8(6), 27-29.

Ruiz, P. A. (2010). Interpretación y difusión: dos formas diferentes de ver el patrimonio. Arqueología y Territorio, 7. https://www.ugr.es/ arqueologyterritorio/PDF7/Parrondo.pdf

Tilden, F. (1977). Interpreting our heritage [La interpretación de nuestro patrimonio] (3. ${ }^{a}$ ed.). https://b-ok.org/book/1081279/9c3473

UNESCO. (2008). Directrices Prácticas para la aplicación de la Convención del Patrimonio Mundial. http://whc.unesco.org/archive/ opguide08-es.pdf

\section{Natalia T. Deza de la Vega}

Historiadora del Programa Municipal para la Recuperación del Centro Histórico de Lima - PROLIMA. Máster en Conservación y Gestión de Bienes Culturales por la Universidad de Salamanca, España. Licenciada en Historia y Diplomada en Ciencias de la Información por la Pontificia Universidad Católica del Perú (PUCP). Se ha desempeñado en puestos de gestión en la Biblioteca Nacional del Perú y el Instituto Geográfico Nacional. Es investigadora-colaboradora en el Grupo de Investigaciones Interdisciplinarias Coloniales del Instituto Riva Agüero de la PUCP. Es autora de cuentos sobre el Centro Histórico de Lima y ha publicado varios artículos históricos sobre esta ciudad. En la actualidad se desempeña como coordinadora del Centro de Documentación e Investigación y del Centro de Interpretación del Centro Histórico de Lima en la Gerencia de PROLIMA.

natalia.deza@pucp.pe

ORCID: http://orcid.org/0000-0003-2739-4540 